\title{
Gamma Knife radiosurgery for brain metastases from pulmonary large cell neuroendocrine carcinoma: a Japanese multi-institutional cooperative study (JLGK1401)
}

\author{
Takuya Kawabe, MD, ${ }^{1,2}$ Masaaki Yamamoto, MD, ${ }^{2}$ Yasunori Sato, PhD, ${ }^{3}$ Shoji Yomo, MD, ,14 \\ Takeshi Kondoh, MD, ${ }^{5}$ Osamu Nagano, MD, ${ }^{6}$ Toru Serizawa, MD, ${ }^{7}$ Takahiko Tsugawa, MD, ${ }^{8}$ \\ Hisayo Okamoto, MD, ${ }^{9}$ Atsuya Akabane, MD, ${ }^{10}$ Kazuyasu Aita, MD, ${ }^{1,11}$ Manabu Sato, MD, ${ }^{11}$ \\ Hidefumi Jokura, MD, ${ }^{12}$ Jun Kawagishi, MD,12 Takashi Shuto, MD, ${ }^{13}$ Hideya Kawai, MD, ${ }^{15}$ \\ Akihito Moriki, MD, ${ }^{16}$ Hiroyuki Kenai, MD, ${ }^{17}$ Yoshiyasu Iwai, MD, ${ }^{18}$ Masazumi Gondo, MD, ${ }^{19}$ \\ Toshinori Hasegawa, MD, ${ }^{20}$ Soichiro Yasuda, MD, ${ }^{21}$ Yasuhiro Kikuchi, MD, ${ }^{22}$ \\ Yasushi Nagatomo, MD, ${ }^{23}$ Shinya Watanabe, MD, ${ }^{2}$ and Naoya Hashimoto, MD'
}

${ }^{1}$ Department of Neurosurgery, Kyoto Prefectural University of Medicine, Graduate School of Medical Science, Kyoto; ${ }^{2}$ Katsuta Hospital Mito Gamma House, Hitachi-naka; ${ }^{3}$ Clinical Research Center, Chiba University Graduate School of Medicine, Chiba; ${ }^{4}$ Saitama Gamma Knife Center, Sanai Hospital, Saitama; ${ }^{5}$ Department of Neurosurgery, Shinsuma Hospital, Kobe; ${ }^{6} \mathrm{Gamma}$ Knife House, Chiba Cardiovascular Center, Ichihara; ${ }^{7}$ Tokyo Gamma Unit Center, Tsukiji Neurological Clinic, Tokyo; ${ }^{8}$ Nagoya Radiosurgery Center, Nagoya Kyoritsu Hospital, Nagoya; ${ }^{9}$ Department of Neurosurgery, Takashima Hospital, Yonago; ${ }^{10} \mathrm{Gamma}$ Knife Center, NTT Medical Center Tokyo, Tokyo; ${ }^{11}$ Kyoto Gamma Knife Center, Rakusai Shimizu Hospital, Kyoto; ${ }^{12 J i r o ~ S u z u k i ~}$ Memorial Gamma House, Furukawa Seiryo Hospital, Osaki; ${ }^{13}$ Department of Neurosurgery, Yokohama Rosai Hospital, Yokohama; ${ }^{14}$ Department of Neurosurgery, Aizawa Hospital, Matsumoto; ${ }^{15}$ Department of Neurosurgery, Research Institute for Brain and Blood Vessels-Akita, Akita; ${ }^{16}$ Department of Neurosurgery, Mominoki Hospital, Kochi; ${ }^{17}$ Department of Neurosurgery, Nagatomi Neurosurgical Hospital, Oita; ${ }^{18}$ Department of Neurosurgery, Osaka City General Hospital, Osaka; ${ }^{19} \mathrm{Gamma}$ Center Kagoshima, Atsuchi Neurosurgical Hospital, Kagoshima; ${ }^{20}$ Department of Neurosurgery, Komaki City Hospital, Komaki; ${ }^{21}$ Department of Neurosurgery, Shiroyama Hospital, Habikino; ${ }^{22}$ Department of Neurosurgery, Southern Tohoku Research Institute for Neuroscience, Southern Tohoku General Hospital, Koriyama; and ${ }^{23}$ Department of Neurosurgery, Kouseikai Takai Hospital, Tenri, Japan

OBJECTIVE In 1999, the World Health Organization categorized large cell neuroendocrine carcinoma (LCNEC) of the lung as a variant of large cell carcinoma, and LCNEC now accounts for $3 \%$ of all lung cancers. Although LCNEC is categorized among the non-small cell lung cancers, its biological behavior has recently been suggested to be very similar to that of a small cell pulmonary malignancy. The clinical outcome for patients with LCNEC is generally poor, and the optimal treatment for this malignancy has not yet been established. Little information is available regarding management of LCNEC patients with brain metastases (METs). This study aimed to evaluate the efficacy of Gamma Knife radiosurgery (GKRS) for patients with brain METs from LCNEC.

METHODS The Japanese Leksell Gamma Knife Society planned this retrospective study in which 21 Gamma Knife centers in Japan participated. Data from 101 patients were reviewed for this study. Most of the patients with LCNEC were men (80\%), and the mean age was 67 years (range 39-84 years). Primary lung tumors were reported as well controlled in one-third of the patients. More than half of the patients had extracranial METs. Brain metastasis and lung cancer had been detected simultaneously in $25 \%$ of the patients. Before GKRS, brain METs had manifested with neurological

ABBREVIATIONS GKRS = Gamma Knife radiosurgery; KPS = Karnofsky Performance Scale; LCNEC = large cell neuroendocrine carcinoma; MET = metastasis; MST = median survival time; NSCLC = non-small cell lung carcinoma; PCI = prophylactic cranial irradiation; RPA = recursive partitioning analysis; RTOG = Radiation Therapy Oncology Group; SCLC = small cell lung carcinoma; SRS = stereotactic radiosurgery; WBRT = whole-brain radiation therapy.

SUBMITTED June 9, 2016. ACCEPTED July 5, 2016.

INCLUDE WHEN CITING DOI: 10.3171/2016.7.GKS161459. 
symptoms in 37 patients. Additionally, prior to GKRS, resection was performed in 17 patients and radiation therapy in 10. A small cell lung carcinoma-based chemotherapy regimen was chosen for 48 patients. The median lesion number was 3 (range 1-33). The median cumulative tumor volume was $3.5 \mathrm{~cm}^{3}$, and the median radiation dose was $20.0 \mathrm{~Gy}$. For statistical analysis, the standard Kaplan-Meier method was used to determine post-GKRS survival. Competing risk analysis was applied to estimate GKRS cumulative incidences of maintenance of neurological function and death, local recurrence, appearance of new lesions, and complications.

RESULTS The overall median survival time (MST) was 9.6 months. MSTs for patients classified according to the modified recursive partitioning analysis (RPA) system were 25.7, 11.0, and 5.9 months for Class 1+2a (20 patients), Class $2 \mathrm{~b}$ (28), and Class 3 (46), respectively. At 12 months after GKRS, neurological death-free and deterioration-free survival rates were $93 \%$ and $87 \%$, respectively. Follow-up imaging studies were available in 78 patients. The tumor control rate was $86 \%$ at 12 months after GKRS.

CONCLUSIONS The present study suggests that GKRS is an effective treatment for LCNEC patients with brain METs, particularly in terms of maintaining neurological status.

http://thejns.org/doi/abs/10.3171/2016.7.GKS161459

KEY WORDS brain metastases; stereotactic radiosurgery; Gamma Knife; lung cancer; oncology; LCNEC; large cell neuroendocrine carcinoma

I N 1999, pulmonary large cell neuroendocrine carcinoma (LCNEC) was categorized as a new histological type of lung cancer and recognized as a variant of large cell carcinoma by the World Health Organization. ${ }^{3}$ LCNEC is an uncommon lung cancer subset, accounting for only $3 \%$ of all lung cancers. ${ }^{8,22,28}$ Since Travis et al. first reported LCNEC in 1991, many authors have reported that LCNECs are aggressive tumors, and patients with these tumors have very poor prognoses. ${ }^{8,12,17,19,22,23,28,29}$ Although LCNEC is categorized among the non-small cell lung carcinoma (NSCLC), the biological behavior of these tumors is very similar to that of small cell lung carcinoma (SCLC). Given the complex clinicopathological and biological features of LCNEC, there is controversy as to whether LCNEC should be treated according to NSCLC or SCLC protocols. Brain metastases (METs) are a common, life-threatening neurological problem for patients with advanced LCNEC, but little information is currently available regarding management of LCNEC patients with brain METs. Prophylactic cranial irradiation (PCI) might be recommended for patients with SCLC, as it prolongs both disease-free and overall survival. ${ }^{2,26} \mathrm{Con}-$ versely, PCI is not administered in patients with NSCLC. ${ }^{11}$ In recent years, stereotactic radiosurgery (SRS) combined with whole-brain radiation therapy (WBRT) has generally been recommended as the first treatment for brain METs. ${ }^{1}$ However, debate persists as to whether WBRT is necessary for all patients with brain METs. The primary argument against WBRT (especially PCI) stems from the risk of deterioration of neurocognitive function. ${ }^{4,5}$ Based on a large cohort originating from a multicenter study conducted by the Japanese Leksell Gamma Knife Society (JLGK1401 study), we aimed to evaluate the efficacy of Gamma Knife radiosurgery (GKRS) for treatment of patients with brain METs from LCNEC.

\section{Methods \\ Patients}

The Japanese Leksell Gamma Knife Society planned this retrospective study. Twenty-one Gamma Knife centers in Japan that treat LCNEC patients with brain METs participated. All centers obtained institutional review board approvals from their own facilities to participate in this study, and their outcome data were then retrospectively combined. Written informed consent was obtained from all patients. This allowed us to examine the data from 101 patients and 387 lesions for this study.

Table 1 summarizes the clinical characteristics of the patients and tumors. The study included 20 women and 81 men. The mean age at the time of radiosurgery was 67 years (range 39-84 years). The mean and median tumor numbers per patient were 4 and 3 (range 1-33). Of the 387 lesions, 304 were located in the supratentorial region and 83 in the infratentorial region. Specifically, 123 lesions were located in the frontal lobe, 77 in the cerebellum, 67 in the parietal lobe, 48 in the temporal lobe, 46 in the occipital lobe, and 26 at other sites. Cumulative tumor volumes ranged from 0.08 to $67.8 \mathrm{~cm}^{3}$ (median $3.5 \mathrm{~cm}^{3}$ ), and the volumes of the largest tumors ranged from 0.04 to 51.5 $\mathrm{cm}^{3}$ (median $2.7 \mathrm{~cm}^{3}$ ).

The primary lung cancer was reported by the referring primary physician to be well controlled in only 33 patients, while 56 also had extracranial METs, i.e., 26 patients had METs in lymph nodes, 15 in bone, 13 pulmonary, 11 hepatic, and so on. Among the 101 patients, tumor presentation was synchronous in 26 and metachronous in the other 75. Thirty-three patients had various neurological symptoms caused by brain METs. The median Karnofsky Performance Scale (KPS) score ${ }^{14}$ at the time of radiosurgery was $90 \%$ (range $40 \%-100 \%$ ). The KPS score was $80 \%$ or better in 85 patients and $70 \%$ or worse in 16 . According to the Radiation Therapy Oncology Group (RTOG) recursive partitioning analysis (RPA) classification system, ${ }^{9} 8$ patients were in RPA Class 1, 84 in RPA Class 2, and 8 in RPA Class 3. Using the modified RPA system, ${ }^{30,31}$ there were 20 patients in Class $1+2 \mathrm{a}, 28$ in Class $2 \mathrm{~b}$, and 46 in Class $2 c+3$. Prior treatments performed at other facilities included surgical removal in 17 patients and WBRT (30 or 40 Gy) in 8 (no patients with PCI). An SCLC-based chemotherapy regimen had been chosen for 48 patients $(70 \%)$. The median irradiation dose at the tumor periphery was 20.0 Gy (range 9.0-28.0 Gy), and the median dose at the tumor center (maximum dose) was 36.0 Gy (range 18.0-50.0 Gy). 
TABLE 1. Summary of clinical characteristics in 101 patients with brain METs from pulmonary LCNEC

\begin{tabular}{|c|c|}
\hline Characteristic & No. (range) \\
\hline \multicolumn{2}{|l|}{ Sex } \\
\hline Women & 20 \\
\hline Men & 81 \\
\hline Mean age in yrs & $67(39-84)$ \\
\hline Mean/median no. of tumors & $4 / 3(1-33)$ \\
\hline Total lesions & 387 \\
\hline \multicolumn{2}{|l|}{ Tumor location } \\
\hline Supratentorial & 304 \\
\hline Infratentorial & 83 \\
\hline \multicolumn{2}{|l|}{ Tumor vol $\left(\mathrm{cm}^{3}\right)$} \\
\hline Median cumulative vol & $3.5(0.08-67.8)$ \\
\hline Median vol of largest lesion & $2.7(0.04-51.5)$ \\
\hline \multicolumn{2}{|l|}{ Primary cancer } \\
\hline Controlled & 33 \\
\hline Not controlled & 59 \\
\hline \multicolumn{2}{|l|}{ Extracerebral METs } \\
\hline No & 37 \\
\hline Yes & 56 \\
\hline \multicolumn{2}{|l|}{ Diagnosis } \\
\hline Synchronous & 26 \\
\hline Metachronous & 75 \\
\hline Median \% KPS score & $90(40-100)$ \\
\hline$\geq 80 \%$ & 85 \\
\hline$<70 \%$ & 16 \\
\hline \multicolumn{2}{|l|}{ Modified RPA class } \\
\hline $1+2 a$ & 20 \\
\hline $2 b$ & 28 \\
\hline $2 c+3$ & 46 \\
\hline \multicolumn{2}{|l|}{ Symptom } \\
\hline No & 64 \\
\hline Yes & 37 \\
\hline \multicolumn{2}{|l|}{ Prior surgery } \\
\hline No & 84 \\
\hline Yes & 17 \\
\hline \multicolumn{2}{|l|}{ Prior WBRT } \\
\hline No & 93 \\
\hline Yes & 8 \\
\hline \multicolumn{2}{|l|}{ Chemotherapy } \\
\hline SCLC based & 48 \\
\hline NSCLC based & 21 \\
\hline Median radiation dose in Gy & $20.0(9.0-28.0)$ \\
\hline
\end{tabular}

\section{Statistical Analysis}

Neurological and neuroimaging evaluations were performed every 2-3 months after the initial GKRS. Overall survival time was defined as the interval between the first SRS for brain METs and death from any cause or the day of the last follow-up. Neurological death was defined as death caused by all intracranial diseases, i.e., tumor recur- rence, carcinomatous meningitis, cerebral dissemination, and progression of other untreated intracranial tumors. Neurological death-free survival time was defined as the interval between the first SRS for brain METs and death from any brain disease or the day of last follow-up.

Control of the GKRS-treated lesion was defined as no remarkable increase, namely regression or unchanged, in tumor diameter. Generally, the criteria for local recurrence were an increased size (over 10\% increase in the maximum diameter) of an enhanced area on postgadolinium T1-weighted MR images and an enlarged tumor core on T2-weighted MR images. ${ }^{13}$ Neurological deteriorationfree survival time was defined as the interval between the first SRS and the day in which any brain disease-caused neurological worsening manifested (that is, local recurrence, progression of new lesions, and SRS-induced complications). In patients with KPS scores of $20 \%$ or less, decreases in scores due to neurological worsening were regarded as events and any others were regarded as censored. Complication-free survival time was defined as the interval until the first SRS-induced complication occurred.

All data were analyzed according to the intentionto-treat principle. For the baseline variables, summary statistics were constructed employing frequencies and proportions for categorical data, and means and standard deviations (SD) for continuous variables. We compared patient characteristics using Fisher's exact test for categorical outcomes and t-tests for continuous variables, as appropriate. For time-to-event outcomes, the time elapsed until a first event was compared using the log-rank test, while the Kaplan-Meier method was used to estimate the absolute risk of each event for each group. Hazard ratios (HRs) and 95\% confidence intervals (CIs) were estimated employing the Cox proportional hazards model. In addition, the cumulative incidences of neurological death, impaired neurological status, and local control failure were estimated employing a competing risk analysis, because death is a competing risk for loss to follow-up..$^{10,25}$

All comparisons were planned, and the tests were 2 -sided. A p value of less than 0.05 was considered to be a statistically significant difference. All statistical analyses were performed by one of the authors (Y.S.), who was not involved in either GKRS treatment or patient follow-up, using SAS software version 9.4 (SAS Institute) and the R statistical program, version 3.10.

\section{Results \\ Overall Survival}

The median post-GKRS follow-up time was 7.2 months (range 2.4-64.0 months) for 26 censored observations, and 75 patients had died as of the end of March 2014. The overall median survival time (MST) after GKRS was 9.6 months (95\% CI 7.8-13.1 months). The Kaplan-Meier plots of all 101 patients are shown in Fig. 1A. Actuarial survival rates were $66.8 \%, 46.0 \%$, and $17.4 \%$ at 6,12 , and 24 months after GKRS, respectively. For patients classified within the modified RPA system, MSTs were 25.7 months (95\% CI 12.4-NA) for patients in class $1+2 \mathrm{a}, 11.0$ months (95\% CI 7.3-14.8) for those in class $2 b$, and 5.9 

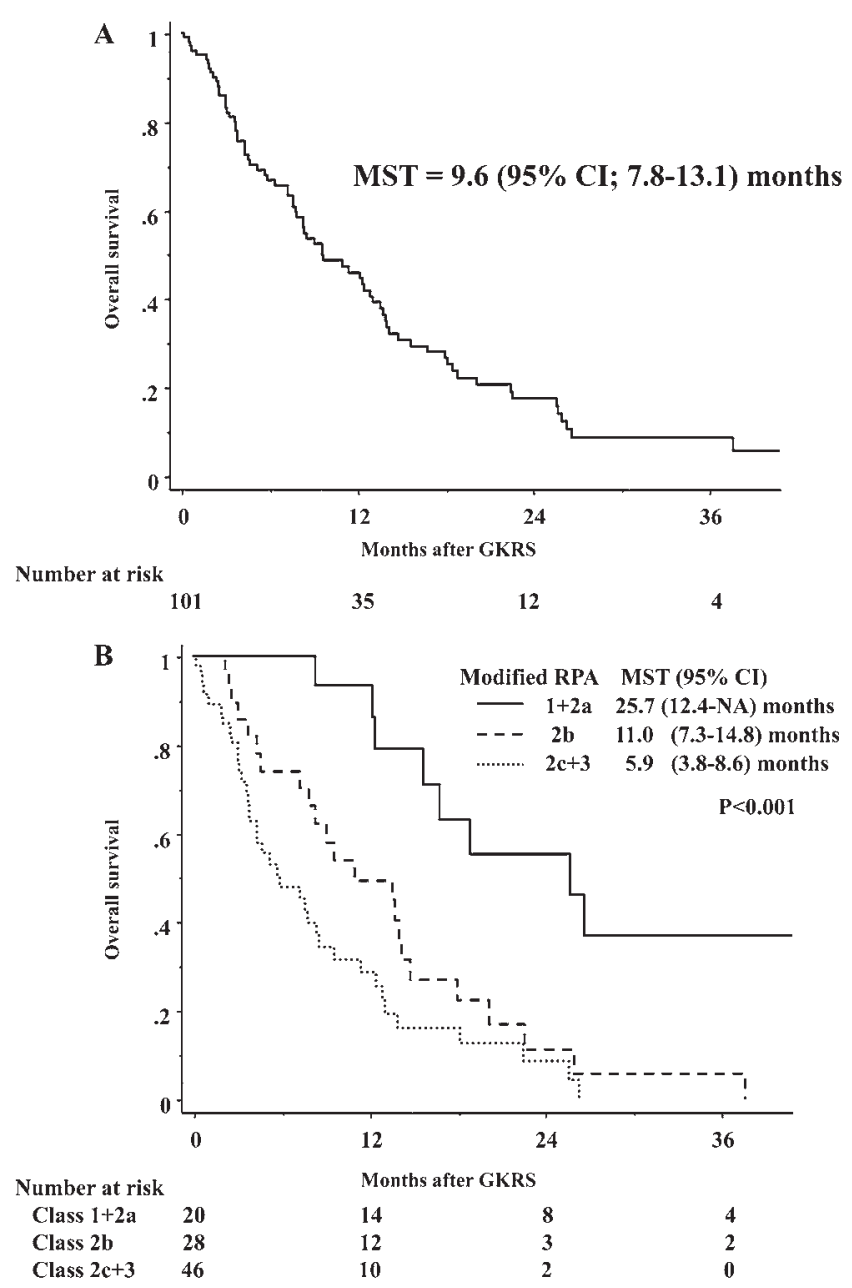

FIG. 1. Overall survival in all 101 patients included in the study (A) and in the patients categorized according to modified RPA classes (B), estimated using the standard Kaplan-Meier method. NA = not available.

months (95\% CI 3.8-8.6) for class 2c+3 (stratified $\mathrm{p}<$ 0.001; Fig. 1B). For chemotherapy selection, i.e., SCLCbased versus NSCLC-based regimen, there was no significant post-GKRS difference in MST between the 2 groups (8.4 months [95\% CI 6.3-11.9] vs 23.4 months [95\% CI 20.3-32.4]; $p=0.905)$. Among the 13 pre-GKRS clinical factors reported (age, sex, histopathological diagnosis, KPS score, number of tumors, cumulative tumor volume, largest tumor volume, peripheral dose, primary tumor status, nonbrain METs, symptoms, prior resection, prior WBRT), multivariate analyses showed 3 clinical factors to be significantly favorable for longer survival: 1) single MET (HR 1.122, 95\% CI 1.051-1.192; $\mathrm{p}=0.001$ ), 2) wellcontrolled primary tumors (HR 2.284, 95\% CI $1.137-$ 4.718; $\mathrm{p}=0.020$ ), and 3) no extracranial METs (HR 2.904, 95\% CI 1.417-6.212; $\mathrm{p}=0.003)$.

\section{Neurological Death-Free Survival}

Among the 75 deceased patients, the cause of death could not be determined in 2 , but was confirmed in the remaining 73: nonbrain disease in 62 (85\%), progression of brain METs in 10 (14\%), and cerebral infarction (no rela- tion to GKRS treatment) in 1 (1\%). In the 62 patients who died due to the primary cancer or nonbrain METs, good brain condition was maintained until 1 to several days before death. Actuarial neurological death rates were 7.4\% and $13.8 \%$ at 12 and 24 months after GKRS, respectively. Among the aforementioned 13 factors, multivariate analyses showed 3 clinical factors to significantly favor longer neurological death-free survival: 1) synchronous MET (HR 52.71, 95\% CI 1.877-6052; $\mathrm{p}=0.018$ ), 2) single MET (HR 1.337, 95\% CI 1.096-1.868; $\mathrm{p}=0.003$ ), and 3) large peripheral dose (HR 1.672, 95\% CI 1.124-3.062; $\mathrm{p}=0.010$ ).

\section{Neurological Deterioration-Free Survival}

After treatment, decreased performance status caused by neurological deterioration occurred in 14 patients (Table 2). Cumulative incidences of neurological deterioration were $13.2 \%$ and $17.5 \%$ at 12 and 24 months after GKRS, respectively (Table 3). Among the aforementioned 13 factors, multivariate analyses showed 3 clinical factors to significantly favor longer neurological deterioration-free survival: 1) better KPS score (HR 1.122, 95\% CI 1.001-1.267; $\mathrm{p}=0.049)$, 2) single MET (HR 1.208, 95\% CI 1.047-1.443; $\mathrm{p}=0.010$ ), and 3 ) extracerebral METs (HR 7.346,95\% CI $1.101-63.292 ; \mathrm{p}=0.039)$.

\section{Follow-Up MRI for Local Tumor Control and New Distant Lesions}

In this series, follow-up MR images were available for 78 patients and 281 lesions. Local recurrence of the treated lesions occurred in 10 patients and 18 lesions, as shown in Table 2. Cumulative incidences of local recurrence were $13.8 \%$ and $13.8 \%$ at 12 and 24 months after GKRS, respectively (Table 3 ). Six of the 10 patients with recurrence underwent additional treatment (a second GKRS procedure in 2, stereotactic radiotherapy in 2, surgical removal in 1 , and a combination of surgical removal and stereotactic radiotherapy in 1), while the other 4 patients received conservative therapies because of their poor systemic conditions. In 3 patients for whom postreatment follow-up MR images were available, these images demonstrated 5 of the treated tumors to be well controlled, while 2 other tumors were not well controlled. None of the 6 clinical factors (tumor volume, peripheral dose, central dose, tumor location [supra- or infratentorial], prior resection, prior WBRT) was found to be statistically significantly associated with a higher recurrence rate on multivariable analyses.

New distant lesions were detected during the postGKRS observation period in 34 patients (Table 2). As shown in Table 3, cumulative incidences of new distant lesion appearance were $45.7 \%$ and $51.9 \%$ at 12 and 24 months after GKRS, respectively. As an additional treatment for new distant lesions, 26 patients underwent a second GKRS procedure, 4 underwent WBRT, and 4 did not have additional treatment. Among the aforementioned 13 factors, multivariable analyses showed 3 clinical factors to be significantly favorable for longer new distant lesion-free survival: 1) male sex (HR 4.660, 95\% CI 1.609-13.075; p $=0.005), 2)$ synchronous MET (HR 5.357, 95\% CI 1.55820.918; $\mathrm{p}=0.007$ ), and 3) single MET (HR 1.136, 95\% CI $1.032-1.266 ; \mathrm{p}=0.011$ ). 
TABLE 2. Treatment results after GKRS: crude incidences

\begin{tabular}{lc}
\hline \multicolumn{1}{c}{ Treatment Result } & No. of Patients (\%) \\
\hline Neurological death & \\
\hline Neurological deterioration & $11(15.1)$ \\
\hline Local recurrence $\dagger$ & $14(13.9)$ \\
\hline New distant lesions $\dagger$ & $10(12.8)$ \\
\hline Repeat GKRS procedure & $34(43.6)$ \\
\hline SRT & $28(27.7)$ \\
\hline WBRT & $3(3.0)$ \\
\hline Surgery & $4(4.0)$ \\
\hline GKRS-related complications & $3(3.0)$ \\
\hline
\end{tabular}

* Based on 73 deceased patients whose cause of death was determined (2 were excluded because cause of death was not available).

$\dagger$ Based on 78 patients ( 23 were excluded because neuroimaging results were not available).

\section{GKRS-Related Complications}

GKRS-related complications occurred in 6 patients (Table 2). One was RTOG Class 1, 3 were RTOG Class 2 (mild transient motor weakness), and 2 were RTOG Class 3 . Of the 2 patients with RTOG Class 3 , one experienced motor weakness 4 months after GKRS and the other experienced intratumoral hemorrhage, eventually necessitating resection 2 days after GKRS. As shown in Table 3, cumulative incidences of GKRS-related complications were $5.5 \%$ and $5.5 \%$ at 12 and 24 months after GKRS, respectively. None of the aforementioned 13 factors was found to be statistically significantly associated with a higher complication rate on multivariable analyses.

\section{Discussion}

\section{Overall Survival and Maintenance of Good Neurological Condition}

Patients with advanced LCNEC generally have poor survival, as seen with all lung cancers. In patients with Stage IV LCNEC, the MST of the entire cohort was reported to be 10.2 months from the time of initial diagnosis. ${ }^{6,16,20,24,27,33}$ Brain METs are a common, life-threatening neurological problem for patients with advanced LCNEC. SRS, which allows radical treatment of brain METs, may have contributed greatly not only to prolonged overall survival but also to maintenance of neurological condition and, ultimately, to reducing the neurological death rate. As Yamamoto et al. reported recently, based on 1194 patients with brain METs treated with GKRS alone, approximately $90 \%$ of patients with brain METs died because of progression of extracranial diseases. ${ }^{32}$ Also, they reported the MST of their lung cancer subset to be 12.5 months $(95 \%$ CI 11.2-13.4): 13.1 months (95\% CI 12.0-14.0) in patients with NSCLC and 8.7 months (95\% CI 7.3-11.5) in patients with SCLC. The post-GKRS MST of the present study, 9.6 months, was slightly worse than that of NSCLC while being slightly better than that of SCLC.

We consider our herein-reported data set, with a relatively large sample size, to show that GKRS has the potential to achieve prolonged maintenance of neurological function and to minimize neurological death for LCNEC
TABLE 3. Treatment results after GKRS*

\begin{tabular}{|c|c|c|c|c|c|}
\hline \multirow[b]{2}{*}{ Treatment Result } & \multicolumn{5}{|c|}{ Cumulative Incidences After GKRS (\%) } \\
\hline & $\begin{array}{c}12 \\
\text { Mos }\end{array}$ & $\begin{array}{c}24 \\
\text { Mos }\end{array}$ & $\begin{array}{c}36 \\
\text { Mos }\end{array}$ & $\begin{array}{c}48 \\
\text { Mos }\end{array}$ & $\begin{array}{c}60 \\
\text { Mos }\end{array}$ \\
\hline Neurological death $\dagger$ & 7.4 & 13.8 & 15.8 & 15.8 & 15.8 \\
\hline Neurological deterioration & 13.2 & 17.5 & 17.5 & 17.5 & 17.5 \\
\hline Local recurrence $\ddagger$ & 13.8 & 13.8 & 16.6 & 16.6 & 16.6 \\
\hline New distant lesions $\ddagger$ & 45.7 & 51.9 & 51.9 & 51.9 & 51.9 \\
\hline GKRS-related complications & 5.5 & 5.5 & 5.5 & 8.4 & 8.4 \\
\hline
\end{tabular}

* Cumulative incidences calculated using a competing risk analysis.

† Based on 73 deceased patients whose cause of death was determined (2 were excluded because cause of death was not available).

$\ddagger$ Based on 78 patients ( 23 were excluded because neuroimaging results were not available).

patients with brain METs, as shown in Table 3. Also, both crude $(5.9 \%)$ and cumulative $(5.5 \%$ at 12 months postGKRS) incidences of GKRS complications were acceptably low as compared with those $(11.0 \%$ and $5.7 \%-8.3 \%$, respectively) of the JLGK0901 study. ${ }^{32}$

\section{Chemotherapy}

The response to chemotherapy is poorer in patients with LCNEC than in those with extensive-stage SCLC. Several studies focusing on LCNEC divided patients into groups receiving NSCLC-based versus SCLC-based chemotherapy regimens. ${ }^{24,27}$ In these series, response rates were better in those administered SCLC-based chemotherapy. However, there is some overlap between NSCLCbased and SCLC-based regimens, which might limit the value of such analyses. Furthermore, our results showed no statistically significant difference between these 2 chemotherapy treatments. In the near future, the role of molecularly targeted agents in treating patients with LCNEC should be assessed.

\section{Is PCI Necessary?}

Naidoo et al. reported on a large group of patients with brain METs: $35 \%$ of patients with Stage IV LCNEC had intracranial lesions at the time of initial diagnosis $(n=$ 17/49), and $12 \%$ had brain METs develop later in the disease course $(n=6 / 49) .{ }^{19}$ The clinical course of patients with SCLC was found to be similar to that of those with LCNEC, i.e., brain METs were detected in up to $18 \%$ of patients at the time of initial diagnosis, and the probability of developing such lesions ranged from $50 \%$ to $80 \%$ in patients who survived for 2 years.$^{15}$ Naidoo et al. suggested that patients with advanced LCNEC may benefit from routine brain-directed surveillance during their disease course and prophylactic therapy similar to that administered for patients with SCLC. ${ }^{19}$ However, nowadays, PCI for the treatment of SCLC is no longer an option, as reported by Nosaki et al. based on a Japanese Phase III study. ${ }^{21}$ In a considerable number of the herein-reported patients, brain METs were detected soon after the initial diagnosis, similar to patients with SCLC. However, salvage treatment with GKRS for new lesions was available and was regarded as being sufficient in most cases. We achieved good neurological death-free and neurological deteriora- 
tion-free survivals without PCI. Therefore, we do not consider it to be necessary to treat LCNEC patients with PCI.

\section{Study Limitations}

The relatively small number of patients and the heterogeneity of the population are limitations of the present study. There might have been a patient selection bias, which would include possible misclassification of LCNEC versus SCLC or other types of NSCLC.,18 Because this study was based on a retrospective chart review at each participating facility, pathological diagnoses were made by a pathologist at each facility, i.e., a systematic pathological review was not performed.

Another possible weakness of this study is that neuroimaging follow-up was lacking in approximately $25 \%$ of patients. However, in most patients in this subset, MRI could not be performed because of early post-SRS death or remarkable deterioration of the patient's general condition, not because the patients were lost to follow-up. As to the low complication rate, the primary physicians who managed our GKRS patients might not have reported relatively minor problems to us. Therefore, an additional weakness of this study is that we may not have surveyed all patients with minor complications.

\section{Conclusions}

To our knowledge, this is the first retrospective multicenter study of patients with brain METs from LCNEC treated with GKRS. Our present study suggests GKRS is an effective treatment for brain METs from LCNEC, particularly in terms of maintenance of neurological status.

\section{Acknowledgments}

We are very grateful to Bierta E. Barfod, Katsuta Hospital Mito Gamma House, for her help with the language editing of this manuscript.

\section{References}

1. Aoyama H, Shirato H, Tago M, Nakagawa K, Toyoda T, Hatano K, et al: Stereotactic radiosurgery plus whole-brain radiation therapy vs stereotactic radiosurgery alone for treatment of brain metastases: a randomized controlled trial. JAMA 295:2483-2491, 2006

2. Aupérin A, Arriagada R, Pignon JP, Le Péchoux C, Gregor A, Stephens RJ, et al: Prophylactic cranial irradiation for patients with small-cell lung cancer in complete remission. $\mathbf{N}$ Engl J Med 341:476-484, 1999

3. Brambilla E, Travis WD, Colby TV, Corrin B, Shimosato Y: The new World Health Organization classification of lung tumours. Eur Respir J 18:1059-1068, 2001

4. Chang EL, Wefel JS, Hess KR, Allen PK, Lang FF, Kornguth DG, et al: Neurocognition in patients with brain metastases treated with radiosurgery or radiosurgery plus whole-brain irradiation: a randomised controlled trial. Lancet Oncol 10:1037-1044, 2009

5. DeAngelis LM, Delattre JY, Posner JB: Radiation-induced dementia in patients cured of brain metastases. Neurology 39:789-796, 1989

6. den Bakker MA, Willemsen S, Grünberg K, Noorduijn LA, van Oosterhout MF, van Suylen RJ, et al: Small cell carcinoma of the lung and large cell neuroendocrine carcinoma interobserver variability. Histopathology 56:356-363, 2010
7. Doddoli C, Barlesi F, Chetaille B, Garbe L, Thomas P, Giudicelli R, et al: Large cell neuroendocrine carcinoma of the lung: an aggressive disease potentially treatable with surgery. Ann Thorac Surg 77:1168-1172, 2004

8. Fasano M, Della Corte CM, Papaccio F, Ciardiello F, Morgillo F: Pulmonary large-cell neuroendocrine carcinoma: from epidemiology to therapy. J Thorac Oncol 10:1133-1141, 2015

9. Gaspar L, Scott C, Rotman M, Asbell S, Phillips T, Wasserman T, et al: Recursive partitioning analysis (RPA) of prognostic factors in three Radiation Therapy Oncology Group (RTOG) brain metastases trials. Int J Radiat Oncol Biol Phys 37:745-751, 1997

10. Gooley TA, Leisenring W, Crowley J, Storer BE: Estimation of failure probabilities in the presence of competing risks: new representations of old estimators. Stat Med 18:695-706, 1999

11. Gore EM, Bae K, Wong SJ, Sun A, Bonner JA, Schild SE, et al: Phase III comparison of prophylactic cranial irradiation versus observation in patients with locally advanced nonsmall-cell lung cancer: primary analysis of radiation therapy oncology group study RTOG 0214. J Clin Oncol 29:272278,2011

12. Iyoda A, Jiang SX, Travis WD, Kurouzu N, Ogawa F, Amano $\mathrm{H}$, et al: Clinicopathological features and the impact of the new TNM classification of malignant tumors in patients with pulmonary large cell neuroendocrine carcinoma. Mol Clin Oncol 1:437-443, 2013

13. Kano H, Kondziolka D, Lobato-Polo J, Zorro O, Flickinger JC, Lunsford LD: T1/T2 matching to differentiate tumor growth from radiation effects after stereotactic radiosurgery. Neurosurgery 66:486-492, 2010

14. Karnofsky DA, Burchenal JH: The clinical evaluation of chemotherapeutic agents in cancer, in Macleod CM (ed): Evaluation of Chemotherapeutic Agents. New York: Columbia University Press, 1949

15. Komaki R, Cox JD, Whitson W: Risk of brain metastasis from small cell carcinoma of the lung related to length of survival and prophylactic irradiation. Cancer Treat Rep 65:811-814, 1981

16. Le Treut J, Sault MC, Lena H, Souquet PJ, Vergnenegre A, Le Caer H, et al: Multicentre phase II study of cisplatinetoposide chemotherapy for advanced large-cell neuroendocrine lung carcinoma: the GFPC 0302 study. Ann Oncol 24:1548-1552, 2013

17. Lo Russo G, Pusceddu S, Proto C, Macerelli M, Signorelli D, Vitali M, et al: Treatment of lung large cell neuroendocrine carcinoma. Tumour Biol 37:7047-7057, 2016

18. Marmor S, Koren R, Halpern M, Herbert M, Rath-Wolfson $\mathrm{L}$ : Transthoracic needle biopsy in the diagnosis of large-cell neuroendocrine carcinoma of the lung. Diagn Cytopathol 33:238-243, 2005

19. Naidoo J, Santos-Zabala ML, Iyriboz T, Woo KM, Sima CS, Fiore JJ, et al: Large cell neuroendocrine carcinoma of the lung: clinico-pathological features, treatment, and outcomes. Clin Lung Cancer [epub ahead of print], 2016

20. Niho S, Kenmotsu H, Sekine I, Ishii G, Ishikawa Y, Noguchi $\mathrm{M}$, et al: Combination chemotherapy with irinotecan and cisplatin for large-cell neuroendocrine carcinoma of the lung: a multicenter phase II study. J Thorac Oncol 8:980-984, 2013

21. Nosaki K, Seto T: The role of radiotherapy in the treatment of small-cell lung cancer. Curr Treat Options Oncol 16:56, 2015

22. Rekhtman N: Neuroendocrine tumors of the lung: an update. Arch Pathol Lab Med 134:1628-1638, 2010

23. Rieber J, Schmitt J, Warth A, Muley T, Kappes J, Eichhorn F, et al: Outcome and prognostic factors of multimodal therapy for pulmonary large-cell neuroendocrine carcinomas. Eur J Med Res 20:64, 2015 
24. Rossi G, Cavazza A, Marchioni A, Longo L, Migaldi M, Sartori $\mathrm{G}$, et al: Role of chemotherapy and the receptor tyrosine kinases KIT, PDGFR $\alpha$, PDGFR $\beta$, and Met in large-cell neuroendocrine carcinoma of the lung. J Clin Oncol 23:87748785,2005

25. Satagopan JM, Ben-Porat L, Berwick M, Robson M, Kutler $\mathrm{D}$, Auerbach AD: A note on competing risks in survival data analysis. Br J Cancer 91:1229-1235, 2004

26. Slotman B, Faivre-Finn C, Kramer G, Rankin E, Snee M, Hatton M, et al: Prophylactic cranial irradiation in extensive small-cell lung cancer. N Engl J Med 357:664-672, 2007

27. Sun JM, Ahn MJ, Ahn JS, Um SW, Kim H, Kim HK, et al: Chemotherapy for pulmonary large cell neuroendocrine carcinoma: similar to that for small cell lung cancer or nonsmall cell lung cancer? Lung Cancer 77:365-370, 2012

28. Travis WD, Linnoila RI, Tsokos MG, Hitchcock CL, Cutler GB Jr, Nieman L, et al: Neuroendocrine tumors of the lung with proposed criteria for large-cell neuroendocrine carcinoma. An ultrastructural, immunohistochemical, and flow cytometric study of 35 cases. Am J Surg Pathol 15:529-553, 1991

29. Varlotto JM, Medford-Davis LN, Recht A, Flickinger JC, Schaefer E, Zander DS, et al: Should large cell neuroendocrine lung carcinoma be classified and treated as a small cell lung cancer or with other large cell carcinomas? J Thorac Oncol 6:1050-1058, 2011

30. Yamamoto M, Sato Y, Serizawa T, Kawabe T, Higuchi Y, Nagano O, et al: Subclassification of recursive partitioning analysis Class II patients with brain metastases treated radiosurgically. Int J Radiat Oncol Biol Phys 83:1399-1405, 2012

31. Yamamoto M, Serizawa T, Sato Y, Kawabe T, Higuchi Y, Nagano O, et al: Validity of two recently-proposed prognostic grading indices for lung, gastro-intestinal, breast and renal cell cancer patients with radiosurgically-treated brain metastases. J Neurooncol 111:327-335, 2013

32. Yamamoto M, Serizawa T, Shuto T, Akabane A, Higuchi Y, Kawagishi J, et al: Stereotactic radiosurgery for patients with multiple brain metastases (JLGK0901): a multi-institutional prospective observational study. Lancet Oncol 15:387-395, 2014

33. Yamazaki S, Sekine I, Matsuno Y, Takei H, Yamamoto N, Kunitoh H, et al: Clinical responses of large cell neuroendocrine carcinoma of the lung to cisplatin-based chemotherapy. Lung Cancer 49:217-223, 2005

\section{Disclosures}

The authors report no conflict of interest concerning the materials or methods used in this study or the findings specified in this paper.

\section{Author Contributions}

Conception and design: Kawabe, Yamamoto. Acquisition of data: all authors. Analysis and interpretation of data: Kawabe, Yamamoto, Y Sato. Drafting the article: Kawabe, Yamamoto, Y Sato. Critically revising the article: Kawabe, Yamamoto, Y Sato. Reviewed submitted version of manuscript: all authors. Approved the final version of the manuscript on behalf of all authors: Kawabe. Statistical analysis: Kawabe, Yamamoto, Y Sato. Administrative/technical/material support: Kawabe, Yamamoto. Study supervision: Yamamoto, Y Sato.

\section{Supplemental Information Previous Prensentations}

This work was presented at the 18th International Meeting of the Leksell Gamma Knife Society, March 15-19, 2016, in Amsterdam, The Netherlands.

\section{Correspondence}

Takuya Kawabe, Kyoto Prefectural University of Medicine Graduate School of Medical Science, 465 Kawaramachi-Hirokoji, Kamigyo-ku, Kyoto 602-8566, Japan. email: taku-626@koto. kpu-m.ac.jp. 